\title{
Fcm - A python library for flow cytometry
}

\author{
Jacob Frelinger ${ }^{\ddagger *}$, Adam Richards ${ }^{\ddagger}$, Cliburn Chan
}

\begin{abstract}
Flow cytometry has the ability to measure multiple parameters of a heterogeneous mix of cells at single cell resolution. This has lead flow cytometry to become an integral tool in immunology and biology. Most flow cytometry analysis is performed in expensive proprietary software packages, and few opensource tool exist for working with flow cytometry data. In this paper we present $f c m$, an BSD licensed python library for traditional gating based analysis in addition to newer model based analysis methods.
\end{abstract}

Index Terms-Flow Cytometry, Model-based Analysis, Automation, Biology, Immunology

\section{Introduction}

Flow cytometry (FCM) has become an integral tool in immunology and biology due to the ability of FCM to measure cell properties at the single cell level for thousands to millions of cells in a high throughput manner. In FCM, cells are typically labeled with monoclonal antibodies to cell surface or intracellular proteins. The monoclonal antibodies are conjugated to different fluorochromes that emit specific wavelengths of light when excited by lasers. These cells are then streamed single file via a capillary tube where they may be excited by multiple lasers. Cells scatter the laser light in different ways depending on their size and granularity, and excited fluorochromes emit light of characteristic wavelengths. Scattered light is recorded in forward and side scatter detectors, and specific fluorescent emission light is recorded into separate channels. Since each fluorescent dye is attached to specific cell markers by monoclonal antibodies, the intensity of emitted light is a measure of the number of bound antibodies of that specificity [Herzenberg2006]. The data recorded for each cell is known as an event, although events may sometimes also represent cell debris or clumps. Modern instruments can resolve about a dozen fluorescent emissions simultaneously and hence measure the levels of a dozen different markers per cell - further increase in resolution is limited by the spectral overlap (spillover) between fluorescent dyes.

Analysis of FCM data has traditionally relied on expert interpretation of scatter plots known as dot plots that show the scattered light or fluorescence intensity for each cell depicted as a point. Expert operators examine these two dimensional dot plots in sequence and manually define boundaries around cell subsets of interest in each projection. The regions demarcated by these boundaries are known as gates, and the cell subsets of interest may require multiple levels of gates to identify. Much work is needed

* Corresponding author: jacob.frelinger@duke.edu

\$ Duke University

Copyright $(02012$ Jacob Frelinger et al. This is an open-access article distributed under the terms of the Creative Commons Attribution License, which permits unrestricted use, distribution, and reproduction in any medium, provided the original author and source are credited.

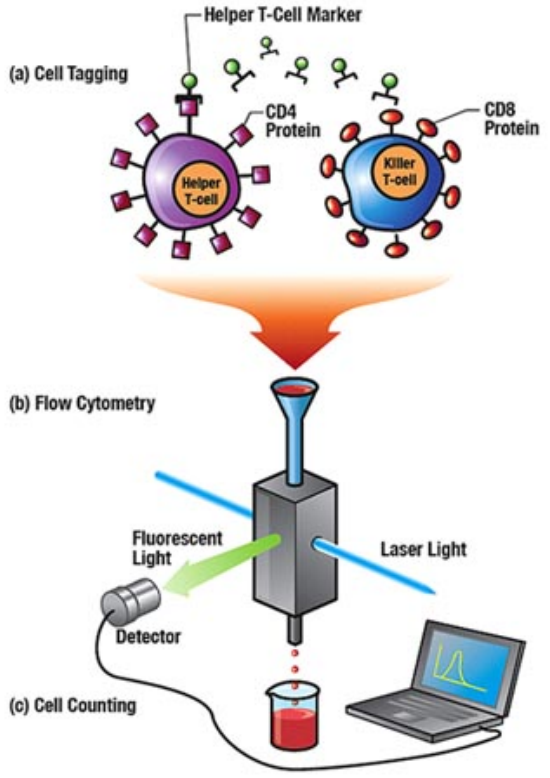

Fig. 1: Diagram of how events are recorded in a flow cytometer provided by lanl.gov

train expert operators to standardize gate placement and minimize variance. Maecker et al [Maecker2005] found a significant source of variability in a multi-center study was due to variability in gating. New technologies have the potential to greatly increase the number of simultaneous markers that can be resolved with FCM. Inductively coupled plasma mass spectrometry [Ornatsky2006] replaces the fluorescent dyes with stable heavy metal isotopes and fluorescent detection with mass spectrometry. This eliminates the spectral overlap (spillover) from fluorescent dyes allowing a significantly increased number of markers to be resolved simultaneously.

With the increasing number of markers that can be resolved simultaneously, there has been an increasing interest in automated methods of cell subset identification. While there is need for such tools, with the exception of the $\mathrm{R}$ BioConductor package, few open source packages exist for doing both traditional analysis and automated analysis. The majority of open source packages simply extract flow events into tabular/csv formats, losing all metadata and providing no additional tools for analysis. $f \mathrm{~cm}$ attempts to resolve this by providing methods for working with flow data in both gating-based and model-based methods.

The goals in writing $f \mathrm{~cm}[\mathrm{fcm}]$ are to provide a generalpurpose python library for working with flow cytometry data. 
Targeted uses include interactive data exploration with [ipython], building pipelines for batch data analysis, and development of GUI and web based applications. In this paper we will explore the basics of working with flow cytometry data using $\mathrm{fcm}$ and how to use fcm to perform analysis using both gating and model based methods.

\section{Loading, compensating and transforming data}

Flow cytometry samples that have been prepared and run through a flow cytometer generate flow cytometry standard (FCS) files, consisting of metadata about the sample, the reagents and instrument used, together with the scatter and fluorescent values for each event captured in the sample acquisition. These binary FCS files are then used to perform quality control and analysis of the data, typically with specialized proprietary software.

In $\mathrm{fcm}$, the loadFCS() function will read in version 2 or 3 FCS files and return a FCMdata object. FCMdata objects contain the recorded scatter and fluorescent marker values for each event in an underlying numpy array, along with the associated metadata stored in the FCS file. In the FCS specification, metdata is stored in separate text, header and analysis sections in the original FCS file, and these can be accessed within a FCMdata instance from FCMdata.notes.text, FCMdata.notes.header, and FCMdata.notes.analysis respectively using either attribute or dictionary lookup conventions. The FCMdata object provides a few methods to directly manipulate the event data extracted from the FCS file, but mostly simply delegates to the underlying numpy array storing the event data matrix. Conveniently, this allows FCMdata objects to perform numpy array methods, such as mean() or std(), and also allows FCMdata objects to be passed to functions expecting numpy arrays. In addition to traditional numpy array indexing, the text names of channels can be used to access channels too.

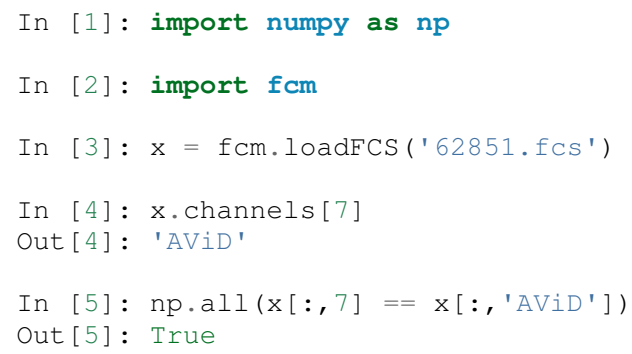

When processing cells and acquiring data, often the emission spectra of fluorescent dyes overlap with neighboring channels. This spillover of light needs to be corrected in a process called compensation that attempts to remove the additional signal from neighboring channels. Using a compensation matrix that describes the amount of spillover from each channel into others, $f \mathrm{~cm}$ will by default apply compensation at the time of loading data, but this default behavior can be suppressed and compensation performed at a later time if necessary. The spillover or compensation matrix is typically found in the FCMdata.notes.text metadata, and loadFCS() will default to compensating using that matrix if another is not specified.

Since FCM fluorescent data typically approximately follows a lognormal distribution, data is often transformed into log or log-like scales. fcm supports both log transforms and logicle [Parks2005] transforms as methods of FCMdata objects. loadFCS() will default to the logicle transform if the data is on the correct scale, that is if P\#R value in the text segment is
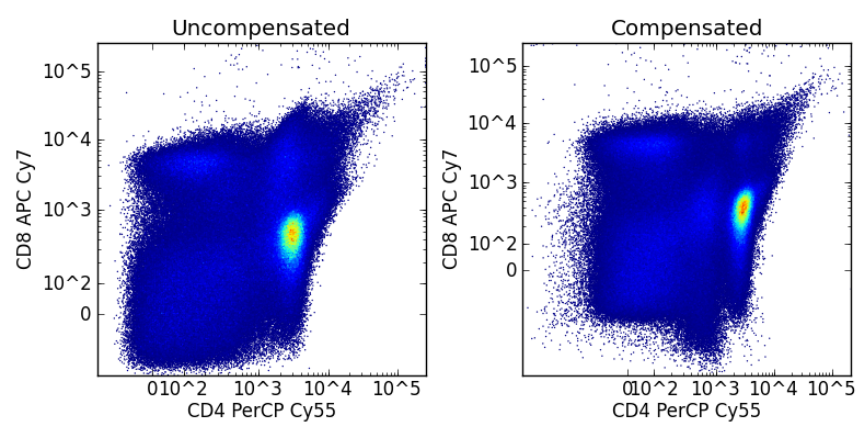

Fig. 2: Compensation changes the data via matrix multiplication operation to reduce the spillover from other markers into each channel and can improve the resolution of individual cell populations.
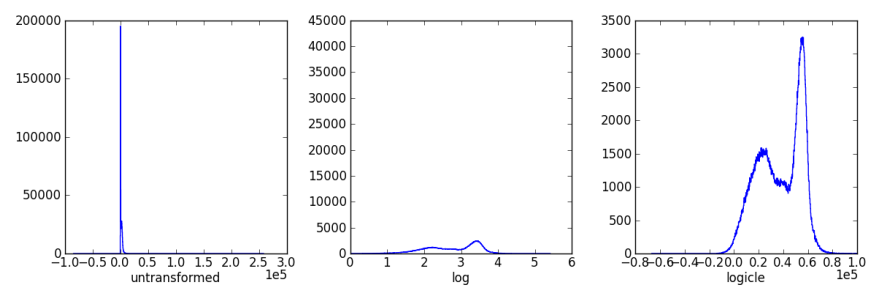

Fig. 3: Illustration of the effects of logicle and log transform on CD3 AmCyan fluorescent from a FCS file from the EQAPOL data set.

262144. Figure 3 illustrates the effects that transforming has on the distribution of events in each fluorescent channel.

\section{Gating Analysis}

In gating based analysis, the objective is to identify specific cellular subsets by sequentially drawing boundary regions, called gates, in a succession of one dimensional and two dimensional plots to select the cellular subsets of interest. Each successive gate captures increasingly specific cellular subsets. Once the required populations have been identified, summary statistics, typically mean or frequency, can easily be computed to compare with other populations.

fcm provides several gating objects to assist in traditional gating analysis of FCS files. Gate objects provided by $\mathrm{fcm}$ include PolygonGate, defining a region of interest by a set of vertices of the boundary of the region, QuadrantGate, dividing a twodimensional projection into four quadrants defined by the point of intersection of all four quadrants, ThresholdGate, a region defined by all points above or below a point in a single parameter, and an IntervalGate, the set of points between two points in a single parameter. In addition to traditional gates, $\mathrm{fcm}$ provides additional gate like filters, DropChannel, to remove unwanted columns from a view, and Subsample, that use a python slice objects to filter events. FCMdata objects gate() method can be used to apply gate objects in successive manner as it returns the updated FCMdata object allowing chaining of gate() calls, like so:

FCMdata.gate (g1) . gate (g2) . gate (g3)

which is equivalent to the following three lines of code:

FCMdata.gate (g1)

FCMdata.gate (g2)

FCMdata.gate (g3)

In fcm, gating FCMdata object does not produce new FCMdata objects, but rather each FCMdata object maintains a tree of 


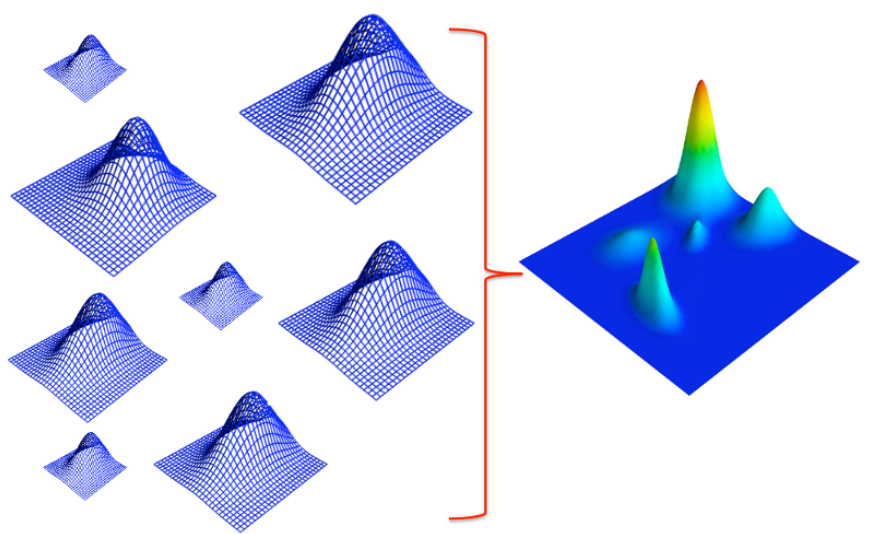

Fig. 4: Mixture models are comprised of multiple simpler distributions. These simpler distributions are added together to describe more complex distributions. Using these simpler distributions, in this case multivariate normal distributions, it becomes possible to describe very complex distributions.

each gated populations. Moving between nodes of the tree, accomplished by the FCMdata.visit() method, selects which events are retured on array lookup, using numpy's efficient indexing to generate views. This allows FCMdata objects to contain an entire analysis in a single object and reduces the need to keep multiple large high dimensional arrays in memory.

\section{Model Based Analysis}

As a result of the increasing dimensionality of FCM data resulting from technological advances, manual analysis is increasingly complex and time-consuming. Therefor there is much interest in finding automated methods of analyzing flow data. Model based analysis is an approach to automate and increase reproducibility in the analysis of flow data by the use of statistical models fitted to the data. With the appropriate multivariate statistical models, data fitting can be naturally performed on the full dimensionality, allowing analysis to scale well with the increasing number of parameters in flow cytometry. Mixture models are one such model based method. Mixture models are often chosen due to their ability to use multiple simpler distributions added together to describe a much more complex distribution as seen in figure 4 .

fcm provides several model based methods for identifying cell subsets, the simplest method being k-means classification, and more advanced methods based on the use of mixtures of Gaussians for data fitting. The general procedure for fitting a data set to a statistical model consists of creating a FCMmodel object containing hyper-parameters, followed by calling its fit method on a collection of (or just one) FCMdata objects to generate ModelResult objects. Each ModelResult object holds the estimated parameters of the statistical model -- a KMeans object representing the centroid locations in a k-means model, or a DPMixture object representing the estimated weights, means and covariances for Gaussian mixture models. These objects can then be used to classify arbitrary datasets or to explore the estimated model parameters.

Gaussian mixture models describe events as coming from a mixture of multiple multivariate Gaussian distributions, where an event $x$ comes from each Gaussian component with probability $\pi_{i}$,

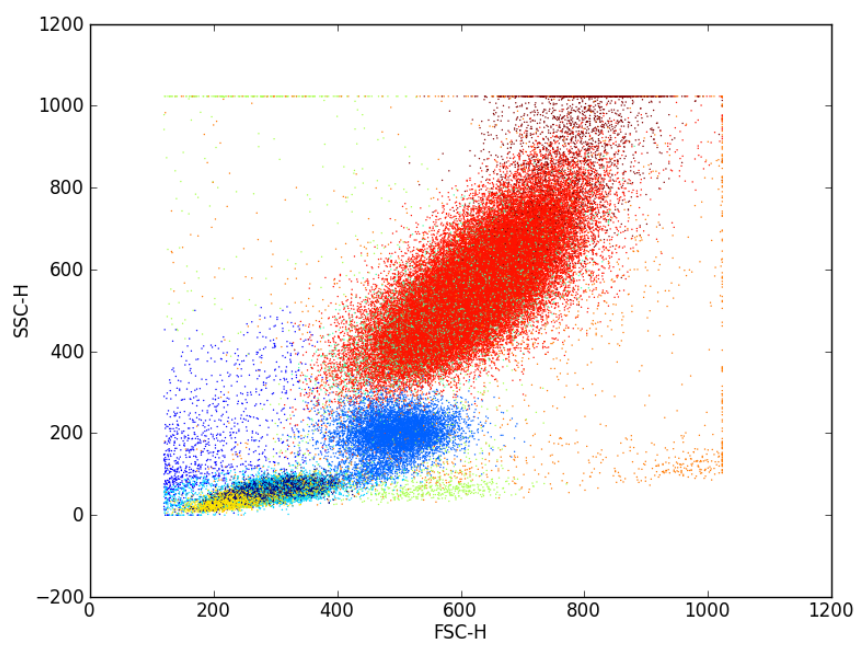

Fig. 5: Events in a sample data set clustered by DPMixtureModel using Bayesian EM

the weight. Hence the overall probability is

$$
p(x \mid \pi, \mu, \sigma)=\sum_{i=1}^{k} \pi_{i} N\left(x \mid \mu_{i}, \sigma_{i}\right)
$$

where $\mathrm{N}$ is a Gaussian, and $x$ can be assigned to the Gaussian component with the highest probability. $f \mathrm{~cm}$ provides two related mixture models to fit data from the [dpmix] package, which is capable of using [gpustats] to utilize GPU cards for efficient estimation of mixture parameters. The two models are DPMixtureModel and HDPMixtureModel, describing a truncated Dirichlet process mixture model, and a hierarchical truncated Dirichlet process mixture model.

DPMixtureModel has two methods of estimating parameters of the model for a given dataset, the first using Markov chain monte carlo (MCMC) and the second using Bayesian expectation maximization (BEM). Sensible defaults for hyperparameters have been chosen that in our experience perform satisfactorily on all FCS data samples we have analyzed.

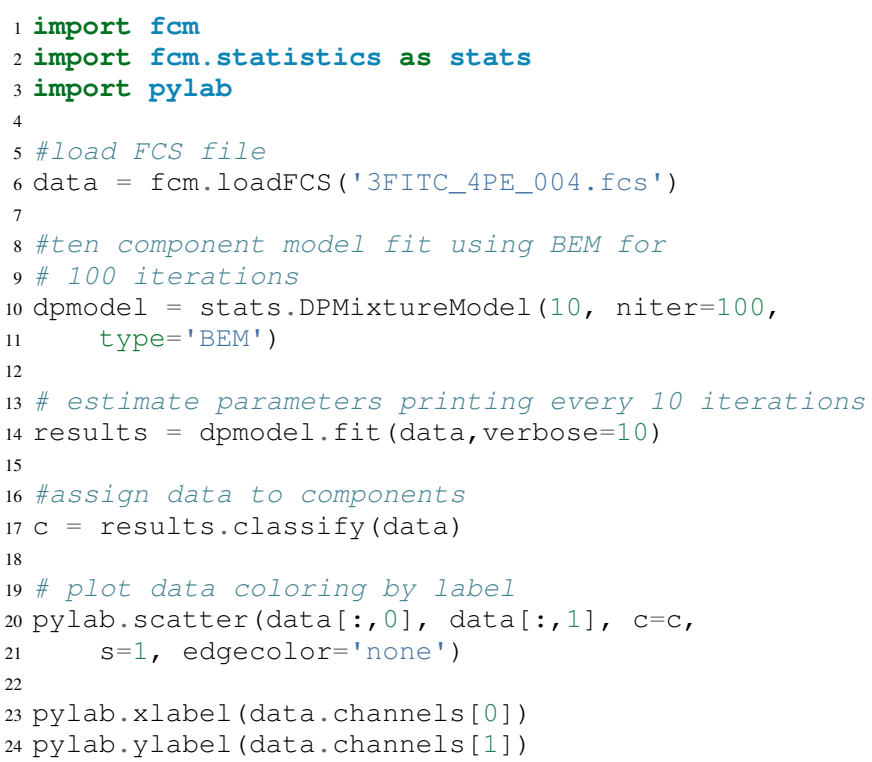

The above code labels each event by color to the cluster it belongs to as seen in figure 5 


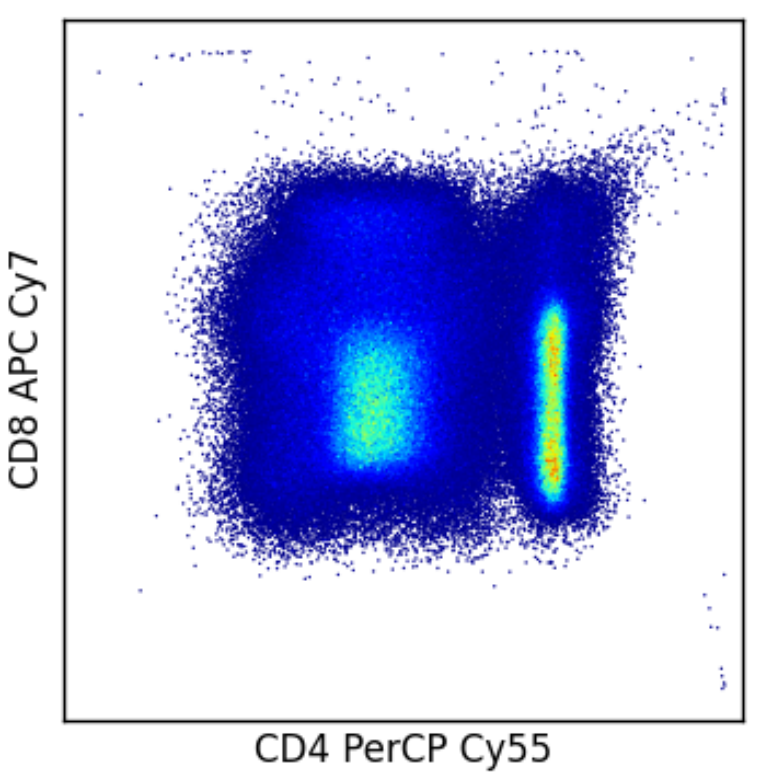

Fig. 6: Pseudo-color heatmap produced by fcm.graphics.heatmap function showing CD4 versus CD8.

HDPMixtureModel fits multiple data sets simultaneously so as to identify a hierarchical model that fits all datasets such that component means and covariance are common to all fitted samples but the weights of components are specific for each sample. Since HDPMixtureModel estimates multiple datasets simultaneously, a list of DPMixture objects is returned corresponding to each of the FCMdata objects passed to HDPMixureMode.fit().

\section{Visualization}

By using packages like [matplotlib] it becomes easy to recreate the typical plots flow cytometry analysts are used to seeing. Convenience functions for several common plot types have been included in the fcm.graphics sub-package. The common pseudocolor dotplot is handled by the function fcm.graphics.pseudocolor()

1 import $\mathrm{fcm}$

2 import fcm.graphics as graph

$3 \mathrm{x}=\mathrm{fcm}$.loadFCS('B6901GFJ-08_CMV pp65.fCS')

4 graph.pseudocolor (x, [('CD4 PerCP Cy55', 'CD8 APC Cy 7')

The above code produces the plot like that seen in figure 6

Another common plot is overlay histograms, which is provided by fcm.graphics.hist()

1 import fom

2 import fcm.graphics as graph

3 from glob import glob

$4 \mathrm{xS}=[\mathrm{fcm}$.loadFCS(x) for $\mathrm{x}$ in glob('B6901GFJ-08_*.fCS')

5 graph.hist (xs, 3, display=True)

The code above will produce the histogram seen in figure 7

More examples of flow cytometry graphics can be seen in the gallery at http://packages.python.org/fcm/gallery.

\section{Conclusion and future work}

Currently fcm is approaching its 1.0 release, providing a stable API for development and we feel $\mathrm{fcm}$ is ready for wider usage

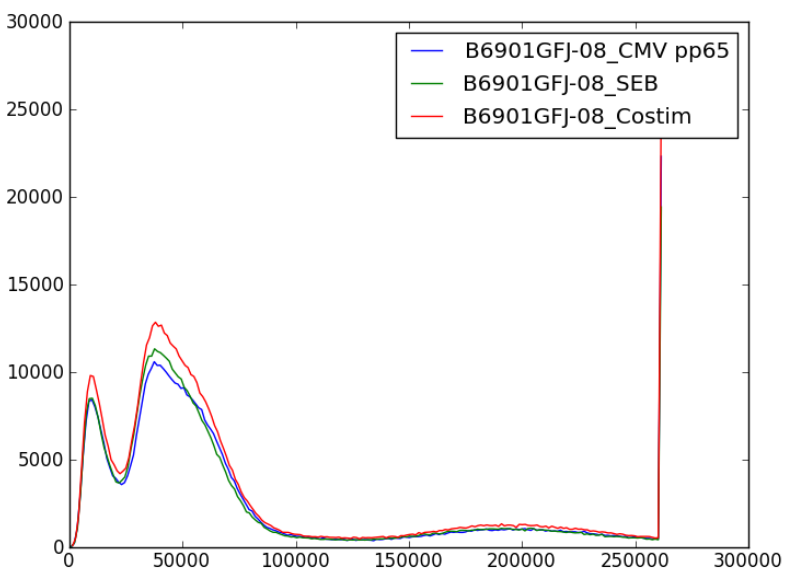

Fig. 7: Overlay histogram of three samples from the EQAPOL data set.

in the scientific community. Internally we use $f \mathrm{~cm}$ for EDA for data sets from HIV/AIDS, caner, and solid-organ transplantation studies. In addition we have developed pipelines for batch analysis of large numbers of FCS files from the Duke Center for AIDS Research, External Quality Assurance Program Oversight Laboratory (EQAPOL), and the Association for Cancer Immunotherapy (CIMT). We have also developed a graphical tool to assist immunologist to perform model based analysis [cytostream]. Our hope is that $\mathrm{fcm}$ can fill a need in the biomedical community and facilitate the growth of python as a tool suited for scientific programming.

With the growing complexity of flow cytometry data, we foresee an increased need for computational tools. Current massspec based flow cytometers are capable of resolving many more parameters than current fluorescent based cytometers, necessitating improved tools for analysis. Imaging cytometers, which take digital images of events as they pass through the detection apparatus, will also produce a wealth of additional information about each event based on analyzing the images generated. These technologies will necessitate improved tools to analyze data generated by these newer cytometers. Our hope is that fcm can meet these needs and continue to grow to address these needs, with specific goals of developing tools to facilitate cross sample comparison and time series of flow data.

]) The next generation of the FCS file standard, Analytical Cytometry Standard, has been proposed, using NetCDF as the format for event storage. The ACS file will be a container allowing storage of much more than the current FCS limitations of event and textual metadata. Thanks to the availability of several good libraries for dealing with NetCDF, and the associated xml and image files proposed to be included in the ACS container, adding support for the finalized version of ACS standard should not be difficult. Gating-ML, an XML format proposed with ACS for describing gates and thier placement, has been gaining popularity. We are exploring how best to implement readers and writers for Gating-ML

\section{Acknowledgements}

We are thankful to Kent Weinhold and the Duke SORF flow core, and the statistics group led by Mike West at Duke University 
for many helpful discussions. Research supported by National Institutes of Health (RC1AI086032-01, UL1RR024128 Cliburn Chan).

\section{References}

[fcm] Frelinger J, Richards A, Chan C, http://code.google.com/ $\mathrm{p} / \mathrm{py}-\mathrm{fcm} /$

[Herzenberg2006] Herzenberg LA, Tung J et al (2006), Interpreting flow cytometry data: a guide for the perplexed, Nat Immunol 7(7):681-685

[Maecker2005] Maecker HT, Frey T et al (2007), Standardization of cytokine flow cytometry assays, BMC Immunol 6:13

[Ornatsky2006] Ornatsky O, Baranov VI et al (2006), Multiple cellular antigent detection by ICP-MS, J Immunol Methods 308(12):68-76

[ipython] Pérez F, Granger BE, IPython: A System for Interactive Scientific Computing, Computing in Science and Engineering, vol. 9, no. 3, pp. 21-29, May/June 2007, doi:10.1109/MCSE.2007.53. URL: http://ipython.org

[Parks2005] Parks, D. R., Roederer, M. and Moore, W. A. (2006), A new "Logicle" display method avoids deceptive effects of logarithmic scaling for low signals and compensated data. Cytometry, 69A: 541-551. doi: 10.1002/cyto.a.20258

[dpmix] Cron A, https://github.com/andrewcron/dpmix

[gpustats] Cron A and McKinney W, https://github.com/dukestats/ gpustats

[matplotlib] Hunter JD, (2007), Matplotlib: A 2D Graphics Environment, Computing in Science \& Engineering 9, 90 (2007)

[cytostream] Richards A, http://code.google.com/p/cytostream/ 